\title{
COMPENSACIÓN DE REACTIVOS EN INSTALACIONES DE SISTEMAS SOLARES FOTOVOLTAICOS PENALIZADAS POR BAJO FACTOR DE POTENCIA
}

\author{
Reactive compensation in solar photovoltaic systems \\ affected by low power factor
}

\author{
Ismael Bautista Almánzar ${ }^{a}$, Miguel Euclides Aybar Mejía ${ }^{b}$, \\ Moisés Blanco ${ }^{c}$, Rommel A. Vicini H. ${ }^{\mathrm{d}}$
}

Recibido: 7/2/2020 • Aprobado: 20/10/2020

\begin{abstract}
Cómo citar: Bautista Almánzar, I., Aybar Mejía, M. E., Blanco, M., \& Vicini H., R. A. (2020). Compensación de reactivos en instalaciones de sistemas solares fotovoltaicos penalizadas por bajo factor de potencia. Ciencia, Ingenierías y Aplicaciones, 3(2), 39-63. Doi: https://doi.org/10.22206/cyap.2020.v3i2.pp39-63
\end{abstract}

\begin{abstract}
Resumen
En este artículo se estudia la posibilidad de compensar potencia eléctrica reactiva (kVAr) en sistemas fotovoltaicos para reducir o anular los recargos causados por bajo factor de potencia, ya que representan un costo adicional en la factura eléctrica. Para compensar la potencia eléctrica reactiva que se consume en los sistemas eléctricos es necesario el uso de elementos que produzcan este tipo de potencia reactiva, por lo que en este estudio se propone un método que compensa la potencia eléctrica reactiva demandada de la red junto al sistema fotovoltaico instalado. El método seleccionado se eligió con base en estudios entre distintas tecnologías en el ámbito de compensación de potencia eléctrica reactiva como inversores, bancos de capacitores, entre otros. Además, se utilizó un analizador de redes eléctricas para registrar el comportamiento de la carga conectada y asi evaluar la efectividad de este método al evaluar las tendencias del consumo de potencia eléctrica reactiva antes y un después de implementar el método seleccionado. El método propuesto es aplicado en una instalación real y su efectividad se respalda con ahorros económicos al mejorar
\end{abstract}

\footnotetext{
a Ingeniería Eléctrica. Instituto Tecnológico de Santo Domingo (INTEC), República Dominicana Correo-e: ismael.bautista@intec.edu.do

${ }^{\mathrm{b}}$ Ingeniería Eléctrica. Instituto Tecnológico de Santo Domingo (INTEC), República Dominicana Correo-e: miguel.aybar@intec.edu.do, ORCID: 0000-0002-4715-3499

c CUCAMA. República Dominicana Correo-e: moisesblanco@gmail.com

d CUCAMA. República Dominicana Correo-e: r.vicini@cucama.do
} 
el factor de potencia y con ahorros ambientales al lograr una reducción de emisiones de dióxido de carbono.

Palabras clave: energía reactiva; factor de potencia; energía solar fotovoltaica; banco de capacitores; compensación de reactivos.

\begin{abstract}
This article studies the possibility of compensating reactive electrical power ( $k V A r)$ in photovoltaic systems to reduce or cancel the surcharges caused by low power factor, since they represent an additional cost in the electricity bill. To compensate for the reactive electric power consumed in electrical systems it is necessary to use elements that produce this type of reactive power, so in this study we propose a method that compensates the reactive electric power demanded from the network next to the system Photovoltaic installed. The selected method was chosen based on studies between different technologies in the field of reactive electric power compensation such as inverters, capacitor banks among others, in addition, an electrical network analyser was used to record the behaviour of the connected load and thus evaluate The effectiveness of this method, when evaluating trends in reactive power consumption before and after implementing the selected method. The proposed method is applied in a real installation and its effectiveness is supported by economic savings by improving the power factor and by environmental savings by achieving a reduction in CO2 carbon dioxide emissions.
\end{abstract}

Keywords: Reactive energy; power factor; photovoltaic solar energy; capacitor bank, reactive compensation. 


\section{Introducción}

Los sistemas de energía eléctrica de corriente alterna son usados en industrias, casas y comercios que tienen equipos que demandan energía; dicha potencia debe ser compensada para mejorar la eficiencia del sistema (Gayatri, Parimi, \& Pavan Kumar, 2018). Además, dicho consumo puede determinar el comportamiento operación de los equipos conectados (ABB, 2016).

En la actualidad, las industrias suelen utilizar sistemas fotovoltaicos para reducir la energía activa $(\mathrm{kWh})$ que se consume de la red eléctrica (Park \& Lappas, 2017; Ben Youssef, Maatallah, Menezo, \& Ben Nasrallah, 2018). En la mayoría de las aplicaciones estos sistemas inyectan en la red potencia activa, con un factor de potencia unitario (Xavier, Cupertino, \& Pereira, 2018), por lo cual estos son incapaces de inyectar reactivos a la red por sí solos.

Estos sistemas mantienen el mismo consumo de energía reactiva (kVArh), debido a que la compensación de potencia reactiva con los inversores de los sistemas fotovoltaicos podría provocar que el convertidor se sobrecargue. Esto ocurre cuando se demanda potencia reactiva simultáneamente al sistema que genera potencia activa nominal (Mulolani, Armstrong, \& Zahawi, 2014), como consecuencia, el factor de potencia de la instalación se reduce considerablemente desde la perspectiva de la red eléctrica.

Estas características hacen que los sistemas fotovoltaicos sean ineficientes desde el punto de vista técnico, ya que al no tener compensación reactiva pueden dar origen a un recargo o penalidad económica dependiendo del país que se esté estudiando. Unos de los elementos asociados al bajo factor de potencias en las industrias es el contenido de los armónicos, los cuales son evaluados y caracterizados en los Estándares IEEE 519 e IEC61000-3, que establecen los límites de contenido armónico; NEC 210-19 FPN No. 4 que define las caídas de voltaje; e IEEE 11592009, que tiene las recomendaciones para el monitoreo de energía.

Según la Superintendencia de Electricidad (2007), en la República Dominicana se aplica una penalidad a las empresas que reciben servicio de las distribuidoras cuyo factor de potencia es inferior a 0.9. Esta 
penalidad corresponde a un costo extra de $1 \%$ de la energía facturada por cada 0.01 en que el factor de potencia baje de 0.90. De acuerdo con Campas \& Martínez (2017) se estipula que un factor de potencia por debajo del valor óptimo causa una gran cantidad de pérdidas eléctricas y puede provocar un problema térmico en los interruptores. Afortunadamente, el factor de potencia se puede controlar con métodos de mejora del factor de potencia diseñados de forma adecuada (Soni \& Panda, 2017).

Eliminar el recargo causado por un bajo factor de potencia mejora la garantía de los resultados de la inversión en renovables (Uğur, Duymaz, Göl, \& Keysan, 2018), por lo que se estudia la posibilidad de reducir la facturación de energía eléctrica para una empresa de servicios logísticos "RODEMSA SAS" al aplicar el modelo propuesto. Otros usuarios del consumo eléctrico han tenido cargos por penalidad por bajo factor de potencia y han presentado ahorros significativos solucionando el consumo de reactivos o factor de potencia (Paulino, 2018).

El objetivo de este estudio es proponer un procedimiento con el que se obtenga una compensación de reactivos de bajo costo en instalaciones fotovoltaicas conectadas a la red eléctrica, dirigido a las pequeńas y medianas industrias de la República Dominicana. Para el desarrollo de este, se estudian los diferentes métodos de compensación de reactivos en sistemas fotovoltaicos (Soni \& Panda, 2017), documentando el funcionamiento de instalaciones fotovoltaicas de inyección a red eléctrica para la compensación de reactivos. Con base en comparaciones, se selecciona un método de compensación de reactivos y se aplica en el caso práctico de una instalación fotovoltaica afectada por cargos por bajo factor de potencia, para poder mostrar los resultados de la implementación y solución de la problemática mediante simulación del sistema.

\section{Metodología}

El estudio de caso fue realizado en una empresa de servicios logísticos "RODEMSA SAS", en la que antes de instalar su sistema solar fotovoltaico se tenía un consumo de energía eléctrica promedio de $20898 \mathrm{kWh}$ para el año 2018, lo que equivale a gastos económicos en la facturación eléctrica promedio de RD\$233000 mensual. Con la finalidad de reducir su consumo de energía eléctrica la empresa instaló un sistema solar foto- 
voltaico de $138 \mathrm{kWp}$ que redujo el consumo promedio de energía eléctrica activa de la empresa en $7770 \mathrm{kWh}$, pero al reducir la magnitud de potencia activa consumida y mantener el consumo de potencia reactiva original se redujo el factor de potencia de la empresa, lo que genera un recargo mensual, dado el bajo factor de potencia, de entre RD \$ 26000 y RD\$ 35 000, según sean los perfiles de carga. La investigación analiza el impacto que tiene la compensación de reactivos en presencia de instalación fotovoltaica y cómo estos consumos de energía reactiva se pueden ver reflejados como recargos de factor de potencia en la facturación, lo que a su vez puede mejorar la garantía de los resultados de la inversión en renovables.

Los autores comparan los diferentes métodos para compensación de reactivos que se pudieran implementar en la empresa para proponer una solución técnico-económica que pudiera ser implementada en la industria. En la figura 1 se presenta la actual configuración potencia instalada en RODEMSA SAS.

En la tabla 1 se presentan distintos métodos de compensación de reactivos propuestos por varios autores, cuya implementación en sistemas eléctricos dependerá del tipo de tecnologías a usar y de los costos asociados a la mismas, además del tiempo de repuesta. En la tabla 2 se comparan los métodos compilados por las investigaciones, donde se destaca que el método que cumple con la mayoría de los criterios evaluados es el banco de compensación automático con filtro desintonizado. En la tabla 3 se presentan algunas observaciones sobre la compensación de reactivos en sistema fotovoltaicos, donde se puede destacar que República Dominicana no cuenta con reglamentación técnica para la implementación de compensación de reactivo mediante los inversores; además, en la misma tabla se resumen la conclusiones que tienen varios autores, que en caso de usar el mismo inversor para compensar potencia reactiva este se debe sobredimensionar, lo cual afecta el tiempo de recuperación de la inversión por el incremento del costo de los mismos.

Se utilizó un analizador de redes AEMC 8336 como se muestra en la figura 2, donde se evidencian las conexiones y el funcionamiento que se hicieron para conectar el sistema eléctrico de estudio (AEMC Instruments, 2016). 


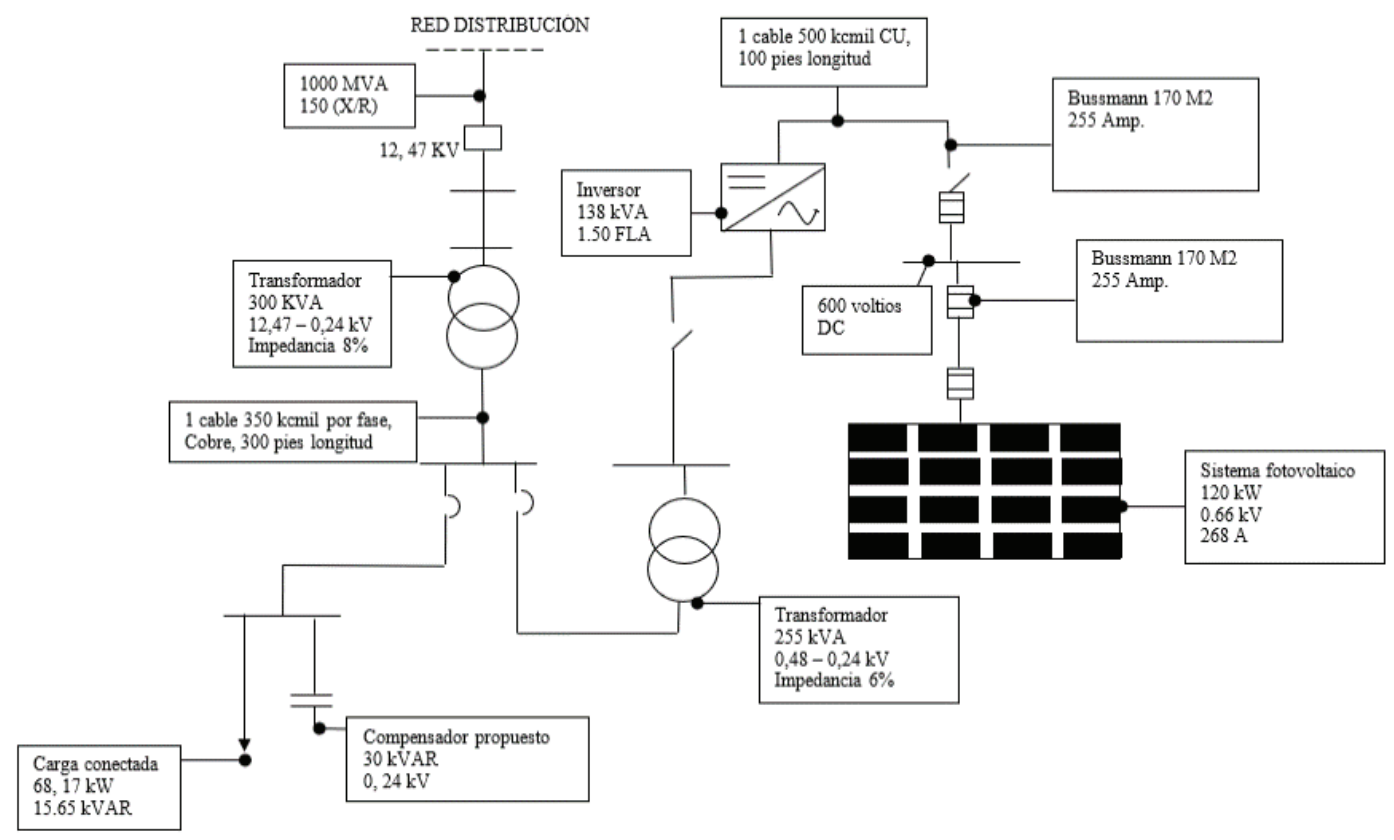

Figura 1. Diagrama unifilar simplificado de la empresa

El analizador se conectó en el tablero principal para monitorear el perfil de carga del almacén. Se utilizó en modo de grabación para poder obtener los datos de potencia activa en $\mathrm{kW}$, potencia aparente en $\mathrm{kVA}$, potencia reactiva en kVAr y factor de potencia, y así poder realizar las simulaciones con los datos reales de la instalación.

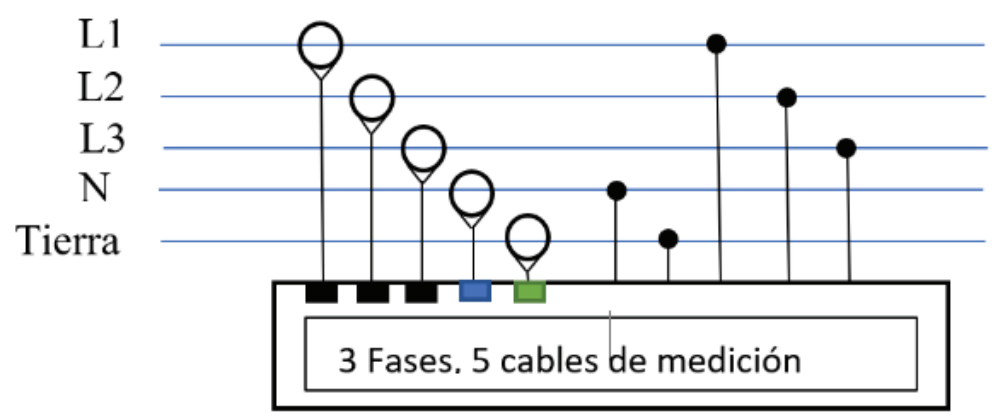

Figura 2. Diagrama de analizador de calidad de energía AEMC 8336 conexión en sistema trifásico. 
De todos los parámetros que se pueden registrar en el instrumento, en este trabajo solo se grafican los de potencia activa $(\mathrm{kW})$, potencia reactiva (kVAr), distorsión armónica (THD) como parámetros de comparación al analizar la mejora que representa la metodología a implementar. En la figura 3 se presenta la potencia activa total antes de la instalación del modelo planteado, de la que se deduce que la operación de la industria no es de 24 horas, con una potencia máxima de $68,18 \mathrm{~kW}$ y un valor mínimo de $7,8 \mathrm{~kW}$ para los períodos fuera de operación. En la figura 4 se presenta la potencia reactiva total antes de la instalación del modelo planteado, donde según los momentos de operación se alcanzan valores máximos de 18,24 kVAr y mínimos de hasta 2,4 kVAr. Tanto la figura 5 como la figura 6 muestran presencia de armónicos en el sistema.

Tabla 1. Métodos para compensación de reactivos

\begin{tabular}{|l|l|}
\hline $\begin{array}{l}\text { Métodos de compensación de } \\
\text { reactivos }\end{array}$ & Estudios realizados \\
\hline $\begin{array}{l}\text { Inversores con capacidad de inyectar } \\
\text { reactivos }\end{array}$ & $\begin{array}{l}\text { (Uğur et al., 2018), (Malatji } \\
\text { \& Chabangu, 2018), } \\
\text { (Gandhi, Rodríguez- } \\
\text { Gallegos, Reindl, \& } \\
\text { Srinivasan, 2018) }\end{array}$ \\
\hline Banco capacitores & (Gandhi et al., 2018) \\
\hline Condensador síncrono & $\begin{array}{l}\text { (Kabir, Mohsin, \& Khan, } \\
\text { 2017) }\end{array}$ \\
\hline Banco de condensadores estáticos & $\begin{array}{l}\text { (Kabir et al., 2017), (ABB, } \\
\text { 2016) }\end{array}$ \\
\hline $\begin{array}{l}\text { Compensadores de Variedad Estática } \\
\text { (SVC) }\end{array}$ & (ABB, 2018). \\
\hline $\begin{array}{l}\text { Banco de condensadores automáticos } \\
\text { con filtros desintonizados }\end{array}$ & $\begin{array}{l}\text { (Espin, 2017), (Sarkar, } \\
\text { Meegahapola, \& Datta, } \\
\text { 2018), (Sarkar et al., 2018) }\end{array}$ \\
\hline
\end{tabular}


Tabla 2. Comparación de métodos para compensación de reactivos

\begin{tabular}{|c|c|c|c|c|c|}
\hline $\begin{array}{l}\text { Criterios/ } \\
\text { Métodos }\end{array}$ & SVC & $\begin{array}{l}\text { Inversor } \\
\text { fotovoltaico }\end{array}$ & $\begin{array}{l}\text { Condensador } \\
\text { síncrono }\end{array}$ & $\begin{array}{l}\text { Condensador } \\
\text { estático }\end{array}$ & $\begin{array}{c}\text { Banco de } \\
\text { condensadores } \\
\text { automáticos } \\
\text { con filtro } \\
\text { desintonizado }\end{array}$ \\
\hline $\begin{array}{l}\text { Capacidad } \\
\text { de absorber } \\
\text { contenido } \\
\text { armónico }\end{array}$ & $\mathrm{x}$ & $x$ & $x$ & & $\mathrm{x}$ \\
\hline $\begin{array}{l}\text { Corrección } \\
\text { sin pasos de } \\
\text { compensación }\end{array}$ & $\mathrm{x}$ & $\mathrm{x}$ & $\mathrm{x}$ & & \\
\hline $\begin{array}{l}\text { Bajo costo de } \\
\text { inversión }\end{array}$ & & & & $\mathrm{x}$ & $\mathrm{x}$ \\
\hline $\begin{array}{l}\text { Bajo costo de } \\
\text { mantenimiento }\end{array}$ & & $x$ & & $x$ & $\mathrm{x}$ \\
\hline $\begin{array}{l}\text { Principal } \\
\text { función } \\
\text { enfocada al } \\
\text { aporte de } \\
\text { reactivos }\end{array}$ & $x$ & & $x$ & $\mathrm{x}$ & $\mathrm{x}$ \\
\hline $\begin{array}{c}\text { Dificultad } \\
\text { de imple- } \\
\text { mentación Baja }\end{array}$ & & & & $\mathrm{x}$ & $\mathrm{x}$ \\
\hline $\begin{array}{l}\text { Alta aplicación } \\
\text { en (FV) }\end{array}$ & & $x$ & & & $x$ \\
\hline Puntuación & 3 & 4 & 3 & 4 & 6 \\
\hline
\end{tabular}


Tabla 3. Observaciones sobre compensación de reactivos en sistema fotovoltaicos

\begin{tabular}{|c|c|}
\hline Autor & Observaciones sobre compensación de reactivos \\
\hline (GSES, 2016). & $\begin{array}{l}\text { Los reactivos que se producen limitarían el ahorro en } \\
\text { la factura eléctrica }\end{array}$ \\
\hline (Sarkar et al., 2018) & $\begin{array}{l}\text { Los requisitos de potencia reactiva se están } \\
\text { convirtiendo en obligatorios. }\end{array}$ \\
\hline $\begin{array}{l}\text { (Organización } \\
\text { Latinoamericana de } \\
\text { Energía, 2013) }\end{array}$ & $\begin{array}{l}\text { Debe existir en el mercado una regulación de reactivos } \\
\text { con energías renovables. }\end{array}$ \\
\hline \multirow{2}{*}{$\begin{array}{l}\text { CNE (Comisión Nacional } \\
\text { de Energía Rep. Dom.) }\end{array}$} & $\begin{array}{l}\text { Existe Ley No. } 57-07 \text { sobre incentivos energía } \\
\text { renovable, pero no existe epígrafe sobre este tema de } \\
\text { compensación de reactivos. }\end{array}$ \\
\hline & $\begin{array}{l}\text { Reglamento de Medición Neta en República } \\
\text { Dominicana el } 28 \text { de junio del 2011, no existe epígrafe } \\
\text { sobre este tema. }\end{array}$ \\
\hline $\begin{array}{l}\text { SIE (Superintendencia de } \\
\text { Electricidad Rep. Dom) }\end{array}$ & $\begin{array}{l}\text { La Resolución SIE-05-2007 metodología cálculo } \\
\text { factor de potencia. La cual se usa para todas las cargas } \\
\text { conectadas a la red de distribución. }\end{array}$ \\
\hline (Cortez Valladares, 2017). & $\begin{array}{l}\text { A las empresas distribuidoras no les conviene que las } \\
\text { industrias manejen un factor de potencia bajo. }\end{array}$ \\
\hline $\begin{array}{l}\text { (Uğur et al., 2018), } \\
\text { (Malatji \& Chabangu, } \\
\text { 2018) }\end{array}$ & $\begin{array}{l}\text { Se debe incrementar el tamańo del inversor para } \\
\text { compensar reactivos. }\end{array}$ \\
\hline (Gandhi et al., 2018) & $\begin{array}{l}\text { Se degrada la vida útil de inversor si se compensa } \\
\text { reactivo por medio de este. }\end{array}$ \\
\hline $\begin{array}{l}\text { (Ndiaye, Xinhui, \& } \\
\text { Agamy, 2015) }\end{array}$ & $\begin{array}{l}\text { El uso de compensadores reactivos en instalaciones } \\
\text { eléctricas minimiza y en algunos casos evita la } \\
\text { necesidad de usar un cambiador de tomas de } \\
\text { transformador para la regulación del voltaje de carga. }\end{array}$ \\
\hline (Mi et al., 2015) & $\begin{array}{l}\text { Las plantas fotovoltaicas ya instaladas podrían ayudar } \\
\text { a la administración de la red mediante equipos de } \\
\text { compensación de reactivos. }\end{array}$ \\
\hline
\end{tabular}

Fuente: elaboración propia. 


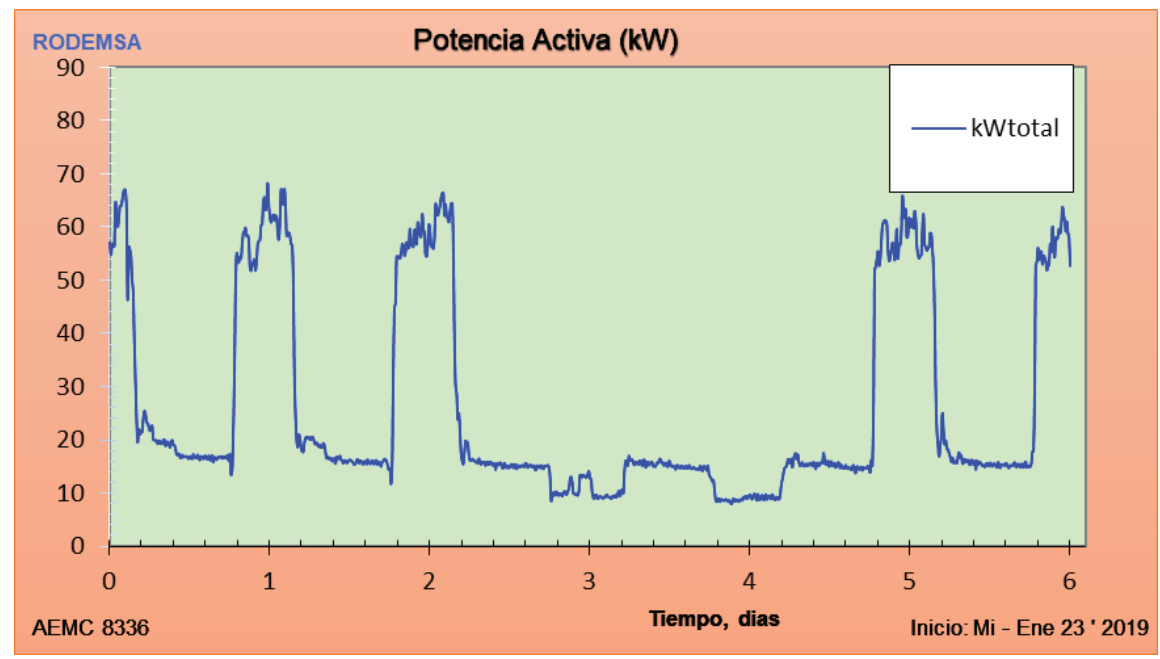

Figura 3. Potencia activa total de la instalación antes de la instalación del modelo planteado

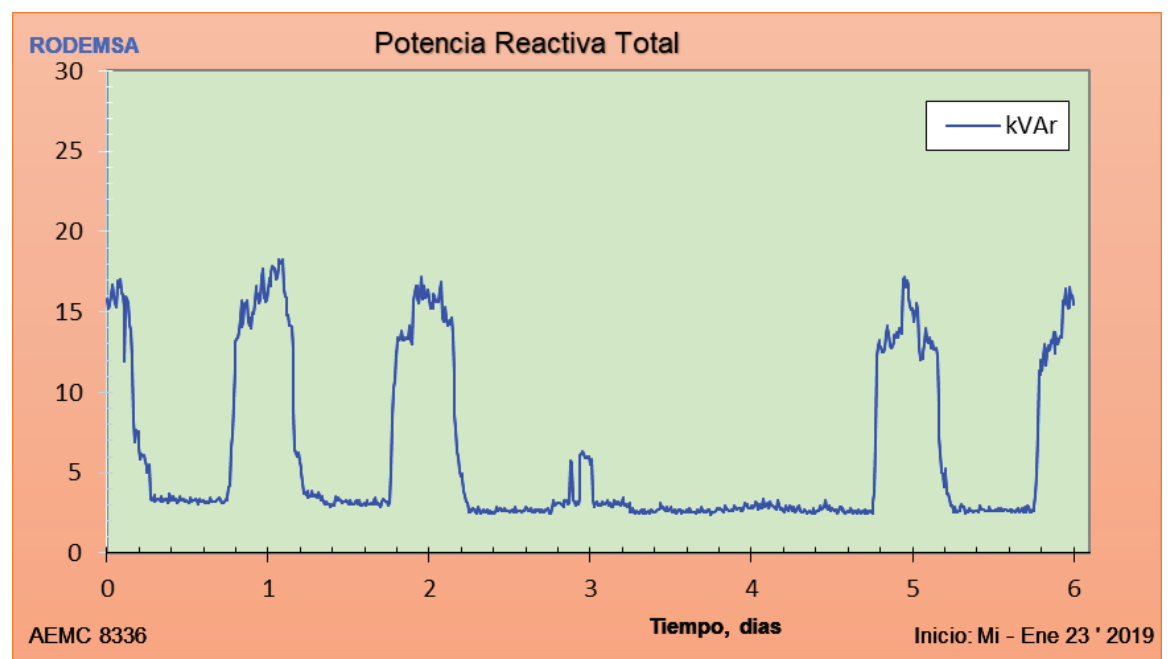

Figura 4. Potencia reactiva total de la instalación antes de la instalación del modelo planteado 


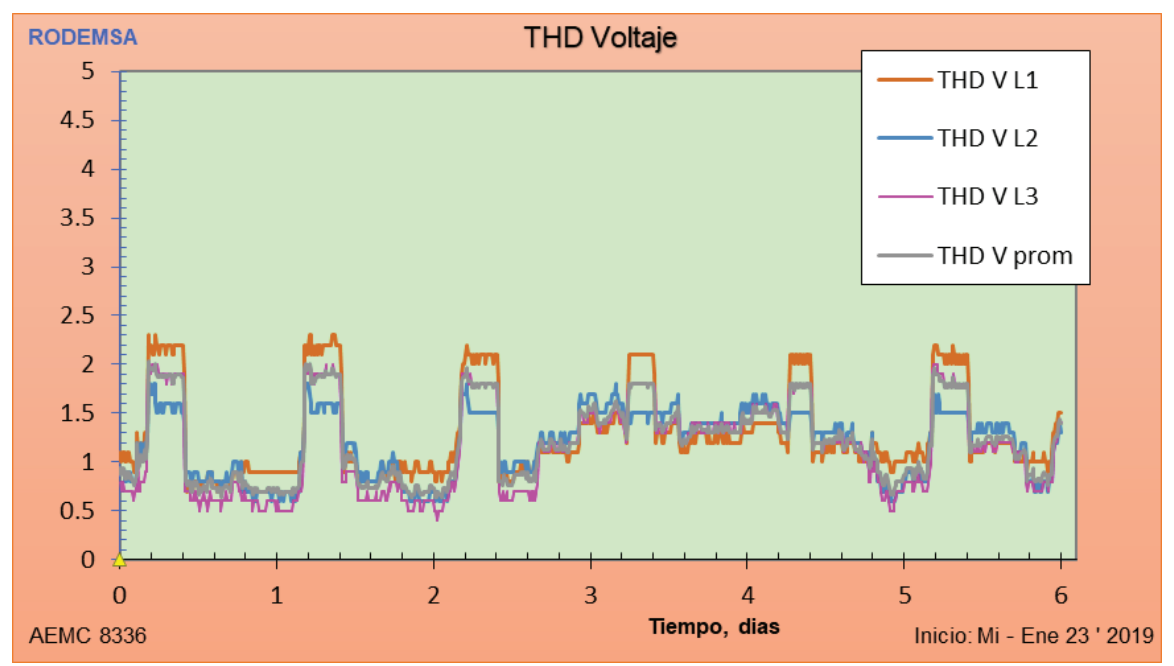

Figura 5. Distorsión armónica de voltaje antes de la instalación del modelo planteado

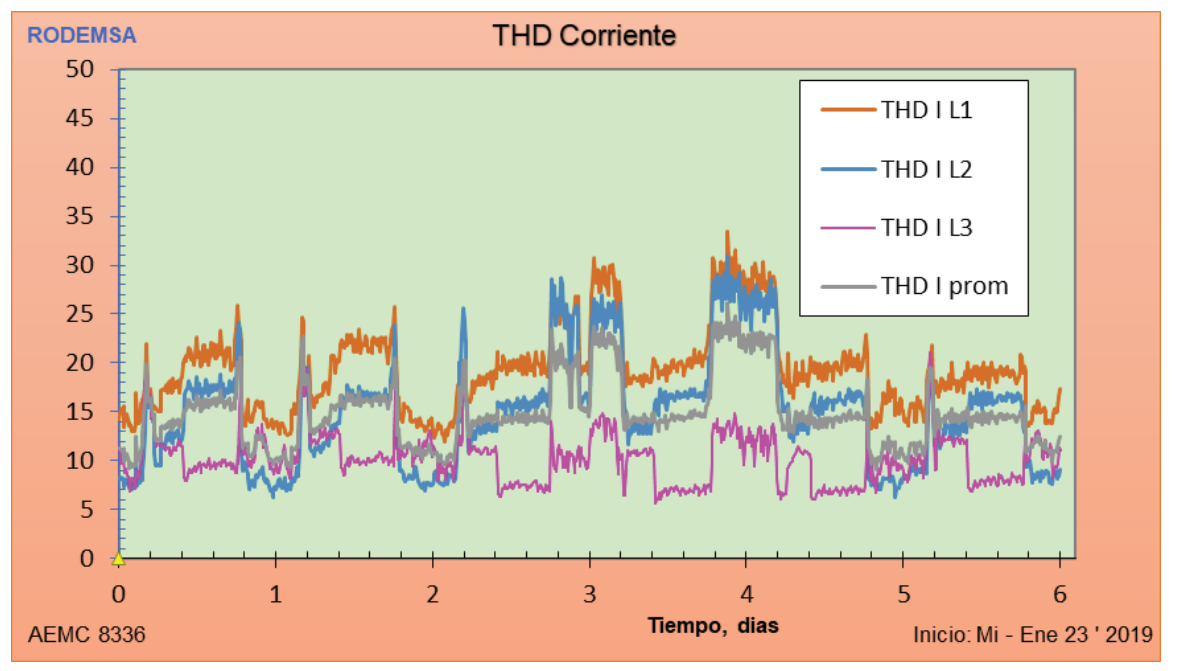

Figura 6. Distorsión armónica de corriente antes de la instalación del modelo planteado 
En trabajos de autores como Vieira et al. (2018), Sayed, El-Ela, y El-Sehiemy, (2016), Norhasmi, Raveendran, y Ramachandaramurthy (2018) se nombran distintos softwares de simulación para modelar y predecir el comportamiento de sistemas eléctricos, entre estos: DIgSILENT, SIMARIS, ETAP, EasyPower. En la misma revisión bibliográfica se determinó que ETAP y EasyPower son los más apropiados para modelar sistemas industriales. De estos dos se seleccionó el software EasyPower porque permite crear modelos simples y ejemplos predeterminados; también permite simular sistemas fotovoltaicos junto a soluciones de sistemas de energía mediante distintos niveles de análisis, además de lo mencionado anterior la versión abierta o gratuita permite simular los elementos del escenario a simular.

\section{Variables}

Para poder realizar una elección correcta con base en las comparaciones de los distintos métodos para compensar reactivos en instalaciones fotovoltaicas, es importante seleccionar los criterios correctos. Los puntos de comparación entre los distintos métodos son: capacidad de absorber contenido armónico, corrección sin pasos de compensación, costo de inversión, costo de mantenimiento, enfoques en aportes de reactivos, dificultad de implementación y aplicación en sistema fotovoltaicos.

Las variables que se tomaron en consideración para este estudio son:

- Potencia activa (Watts).

- Potencia reactiva (VAr).

- Factor de potencia (f.p.)

- Frecuencia eléctrica $(\mathrm{Hz})$.

- Voltaje (V).

\section{Simulación e implementación}

Con la intención de garantizar la vida útil del inversor y que se mantenga produciendo energía activa, con base en sus características y el nivel de inversión de los métodos estudiados se elige el banco de compensación automático con filtro de rechazo. 
Al analizar los perfiles de carga se determinó que la propuesta consiste en banco de compensación automático de $30 \mathrm{kVAr}$ a $240 \mathrm{VAC}$ con filtros de rechazo, equivalente a $23 \mathrm{kVAr}$ en $208 \mathrm{VAC}$ a $60 \mathrm{~Hz}$. Se propone compensar con $30 \mathrm{kVAr}$ para evitar recargo y respaldar el sistema para un crecimiento futuro (promedio esperado es de 0.98 ).

Los armónicos detectados en la figura 5 y la figura 6 indican que debe de aplicarse un filtro de rechazo para evitar la amplificación armónica que pueda ocasionar daño futuro en los equipos. Utilizando el comando Filter Tuning de Microsoft Excel se calculan los parámetros del filtro a utilizar con base en las ecuaciones siguientes:

Reactancia inductiva expresada en $\mathrm{Ohm}$.

$$
\mathrm{Xl}=2 \pi \mathrm{f} \mathrm{L}
$$

Inductancia reactor expresada en Henry

$$
L=\frac{X l}{2 \pi f}
$$

Reactancia capacitiva expresada en Ohm.

$$
\mathrm{Xc}=\frac{1}{2 \pi \mathrm{fC}}
$$

Capacitancia expresada en Faradio.

$$
C=\frac{1}{2 \pi f X c}
$$

Orden del armónico.

$$
\mathrm{h}=\sqrt{\frac{1}{\text { Factordesintozinacion } \%}}
$$


Frecuencia de resonancia.

$$
f r=\frac{1}{2 \pi \sqrt{L C}}
$$

Capacitancia expresada en Ohm.

$$
\mathrm{Xc}=\frac{\mathrm{k} V^{2}}{\mathrm{MVAr}}
$$

Los resultados obtenidos de los cálculos fueron:

- Factor de sintonización $7 \%$.

- $\mathrm{h}=3,7797$ el orden del armónico

- $\mathrm{Xc}=3,5267 \Omega$ reactancia capacitiva

- $\quad \mathrm{C}=0.752 \mathrm{mF}$ capacitancia

- $\quad \mathrm{fr}=227 \mathrm{~Hz}$ frecuencia

- $\quad$ Xc en $\mathrm{fr}=0.933 \Omega$

- $\quad \mathrm{L}=0.655 \mathrm{mH}$ inductancia

- $\mathrm{Xl}$ en $\mathrm{fr}=0,2469 \Omega$. Reactancia inductiva

La salida de potencia reactiva con el reactor desintonizado con la configuración propuesta es de aproximadamente de $13.19 \mathrm{kVAr}$ en voltaje 208 VAC.

La ubicación del módulo de compensación de reactivos se seleccionó con base en los puntos que utilizaban los distintos autores en sus investigaciones, y se muestra en la figura 7.

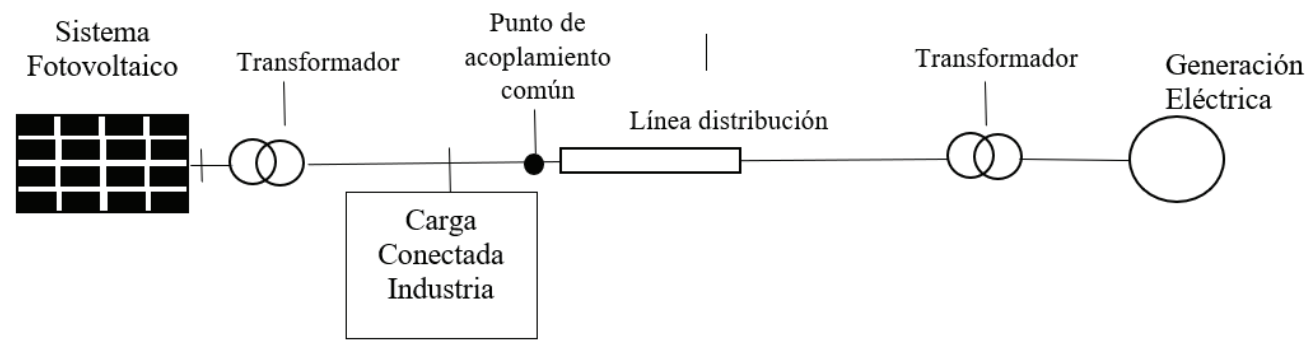

Figura 7. Ubicación del módulo de compensación de reactivos en el punto común de acoplamiento (PCC) 
Con todos los parámetros medidos se procede a la simulación en el software EasyPower para el funcionamiento del sistema con la implementación del método.

El módulo automático de compensación está compuesto por 2 capacitores 15 kVAr 240 V 60 Hz Marca: Circutor, 2 reactores para $15 \mathrm{kVAr}$ $240 \mathrm{~V} 3$ fases $7 \%$, 2 interruptores termo magnético riel din 50 A 3 polos Marca: Schneider, 1 borne de interconexión Marca: Schneider, 1 sensor de alto y bajo voltaje secuencia y pérdida de fase, 2 contactores magnéticos para $15 \mathrm{kVAr} 240 \mathrm{~V}$, luz piloto con indicación de corriente para cada etapa, analizador de redes eléctricas Marca: Circutor Modelo: CVM C10, el cual está programado para medir la reactiva, e ir entrando las etapas según necesidad y 3 transformadores de corriente núcleo partido Marca: Winston relación 800:5. Con compensación de $30 \mathrm{kVAr}$ (en dos etapas de $15 \mathrm{kVAr}$ ), se evita recargo (<0,9 se tiene recargo), el promedio esperado será de 0,97 .

\section{Caracterización}

La instalación posee tres transformadores monofásicos con una potencia de $100 \mathrm{kVA}$ cada uno, una relación de transformación de 12,470/240V e impedancia (Z) de 4,4\%. Dichos transformadores son de tipo poste y se encuentran conectados en delta-estrella. Para la conexión del sistema fotovoltaico al sistema se tiene un transformador trifásico seco con una potencia de $225 \mathrm{kVA}$ que trabaja a $480 / 208 \mathrm{~V}$ en conexión delta-estrella.

La instalación se encuentra ubicada en la Carretera Sánchez, km 13 Haina Oriental, Santo Domingo, R.D. Posee una potencia instalada: $138 \mathrm{kWp}$, la cual fue puesta en servicio el 15/09/2018 y una superficie de módulos: $775 \mathrm{~m}^{2}$. En la tabla 4 se muestran las características de los inversores fotovoltaicos de la instalación. Donde se puede observar que la característica esencial de cada uno es su potencia, ya que son del mismo modelo y poseen conexiones similares. 
Tabla 4. Características del inversor 1 e inversor 2

\begin{tabular}{|l|l|l|}
\hline Designación: & Inversor 1 & Inversor 2 \\
\hline Potencia & $68.31 \mathrm{kWp}$ & $69.69 \mathrm{kWp}$ \\
\hline Área $\left(m^{2}\right):$ & 383.79 & 391.55 \\
\hline $\begin{array}{l}\text { Tipo de } \\
\text { inversor: }\end{array}$ & Huawei SUN2000-65KTL-M0 & Huawei SUN2000-65KTL-M0 \\
\hline $\begin{array}{l}\text { Tipo de } \\
\text { módulos: }\end{array}$ & LONGI Solar LR6-72-345M/ & LONGI Solar LR6-72-345M/ \\
\hline $\begin{array}{l}\text { Número de } \\
\text { módulos: }\end{array}$ & 198 & 202 \\
\hline $\begin{array}{l}\text { Ocupación de } \\
\text { las entradas: }\end{array}$ & $1 / 1 / 1 / 1 / 1 / 1$ & $1 / 1 / 1 / 1 / 1 / 1$ \\
\hline $\begin{array}{l}\text { Conectado en } \\
\text { serie: }\end{array}$ & $18 / 18 / 18 / 18 / 18 / 18$ & $18 / 18 / 18 / 18 / 19 / 19$ \\
\hline $\begin{array}{l}\text { Conectado en } \\
\text { paralelo: }\end{array}$ & $2 / 2 / 2 / 2 / 2 / 1$ & $2 / 2 / 2 / 1 / 2 / 2$ \\
\hline
\end{tabular}

\section{Análisis económico}

Siecker, Kusakana y Numbi (2018) presentan indicadores de desempeño económico como: el período de recuperación simple (SPP), la tasa interna de rendimiento (IRR), la relación de beneficios a costo (BCR) y el costo del ciclo de vida (LCC), pero afirma que el método SPP es fácil de entender y calcular. Sin embargo, este método no tiene en cuenta que el dinero se devalúa con el tiempo debido a la inflación y otros factores. Además, la vida útil del proyecto no se tiene en cuenta con este método, donde los inversionistas no serán plenamente conscientes de la rentabilidad del proyecto.

Implementar el modelo desarrollado en esta investigación para la compensación de reactivos en sistemas fotovoltaicos tiene un costo aproximado de $\mathrm{RD} \$ 160$ 000, y al aplicar este método se ahorran unos $\mathrm{RD} \$ 28871$ correspondientes a la sanción que se evita al elevar el factor de potencia a valores óptimos. Se utiliza la ecuación 8 para utilizar el método de retorno simple y así calcular los meses en los que se recupera la inversión, pero teniendo en cuenta las limitaciones mencionadas anteriormente. El cálculo realizado determinó que la recuperación de la inversión se realiza en menos de 6 meses. 


\section{Análisis ambiental}

Al instalar la propuesta de compensación de reactivos con el uso de capacitores se mantiene la misma generación de energía activa $(\mathrm{kWh})$ de la planta fotovoltaica, por lo que se puede asumir como si se estuviera evitando generar esa energía con tecnologías que producen con base en combustibles fósiles.

Según la bibliografía consultada: si se quiere destinar el inversor a compensar factor potencia SMA, es conveniente sobredimensionarlo en un $20 \%$ para mantener el margen de energía activa producida, por lo que el dedicar el inversor a compensar factor de potencia sin sobredimensionarlo reducirá su producción de energía eléctrica en un $20 \%$.

La reducción de emisiones se calcula al comparar la producción con las tecnologías que funcionan en base a carbón, independientemente de la matriz energética de la República Dominicana. Para calcular la reducción de las emisiones de $\mathrm{CO}_{2}$ se toma un factor de $0.9 \mathrm{~kg}$ de $\mathrm{CO}_{2}$ por cada kWh.

Tomando los valores de generación fotovoltaica del sistema propuesto, asumiendo además el $20 \%$ de su producción, el cual sería de $3335 \mathrm{kWh}$, y aplicando el factor de $0.9 \mathrm{~kg} / \mathrm{kWh}$, se obtuvo un consumo evitado de $\mathrm{CO}_{2}$ de $3002 \mathrm{~kg} \mathrm{CO} /$ mes lo que al año se proyecta en $36018 \mathrm{~kg}$ $\mathrm{CO}_{2} /$ ańo, lo cual es un impacto positivo, ya que alienado al objetivo 13 (acción por clima) y objetivos 7 (energía asequible y no contaminantes) de los Objetivos de Desarrollo Sostenibles (ODS).

\section{Resultados}

Luego de implementar el modelo se repiten las mediciones con el analizador de potencia. En la figura 8 se muestra el comportamiento de la potencia activa de la instalación, la cual no difiere mucho del comportamiento mostrado en la figura 3, evidenciando que el perfil de carga es muy estable. El valor máximo de potencia es $66.81 \mathrm{~kW}$ y el valor mínimo es $7.65 \mathrm{~kW}$ en los momentos fuera de operación. 


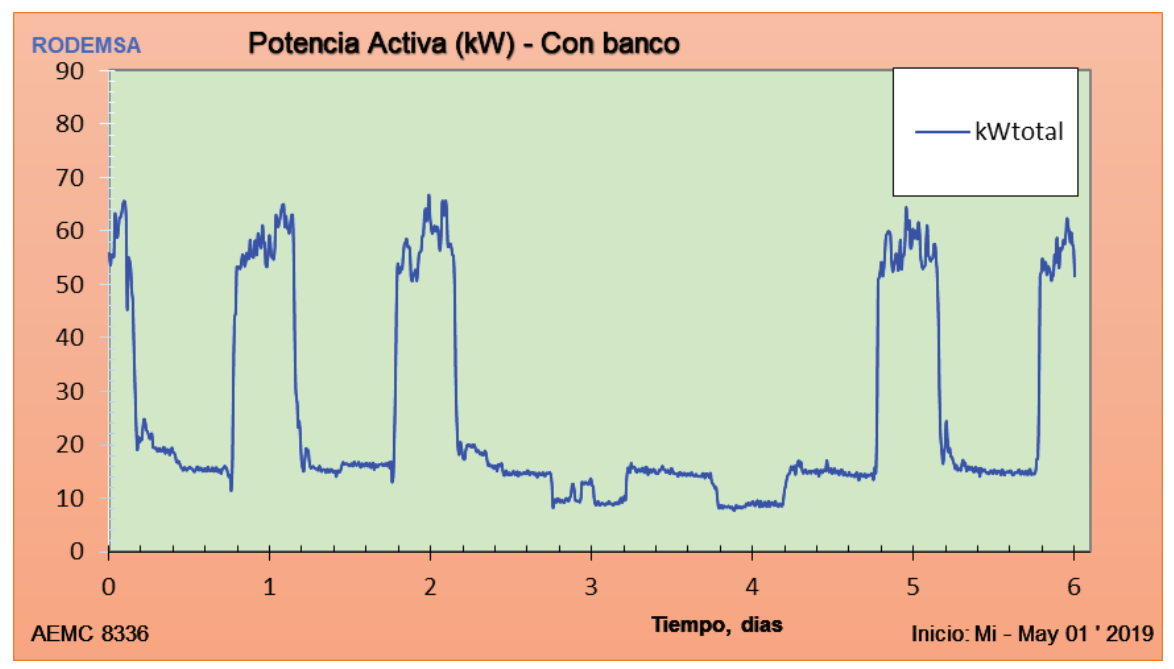

Figura 8. Potencia activa total de la instalación post implementación

En la figura 9 se muestra el comportamiento de la potencia reactiva de la instalación, donde se pueden observar cambios de la demanda de reactivos con una reducción de la demanda a la red entre los $18.24 \mathrm{kVAr}$ mostrados en la figura 4 y los $1.82 \mathrm{kVAr}$ obtenidos después de la implementación.



Figura 9. Potencia reactiva total de la instalación post implementación 
En la figura 10 y la figura 11 se muestra la distorsión armónica del voltaje y la corriente, respectivamente. La distorsión armónica total (THD) promediada del voltaje es de $1.21 \%$, con una THD máxima de $1.8 \%$. En el caso de la corriente, la distorsión armónica total (THD) promediada es de $13.96 \%$, con un THD máximo de $23.1 \%$, para el voltaje la distorsión armónica total se encuentra dentro del límite recomendado que es $5 \%$.



Figura 10. Distorsión armónica de voltaje post implementación



Figura 11. Distorsión armónica de corriente post implementación 
$\mathrm{Al}$ aplicar el método seleccionado se realizaron nuevas mediciones para contrastar los resultados antes y después de aplicar el sistema. En la tabla 5 se presentan los parámetros eléctricos antes de aplicar el banco de compensación de reactivos, mientras que la tabla 6 muestra los parámetros eléctricos luego de aplicarlo.

Tabla 5. Parámetros eléctricos sin aplicar el banco de compensación de reactivos

\begin{tabular}{|c|c|c|c|}
\hline FACTURACIÓN MAYO 2019 & $\begin{array}{c}\text { Energía } \\
\text { Activa } \\
(\mathrm{kWh})\end{array}$ & $\begin{array}{l}\text { Energía } \\
\text { Reactiva } \\
\text { (kVArh) }\end{array}$ & $\begin{array}{l}\text { Factor de } \\
\text { potencia }\end{array}$ \\
\hline Energía demandada Red Distribución & 24405 & 18500 & 0,8 \\
\hline Energía generada planta FV & 16675 & 0 & 1 \\
\hline $\begin{array}{l}\text { Energía generada banco } \\
\text { compensación }\end{array}$ & - & - & - \\
\hline Energía resultante & 7730 & 18500 & 0.39 \\
\hline Penalidad & $51 \%$ & & \\
\hline
\end{tabular}

Tabla 6. Parámetros eléctricos luego de aplicar el banco de compensación de reactivos

\begin{tabular}{|c|c|c|c|}
\hline FACTURACIÓN MAYO 2019 & $\begin{array}{c}\text { Energía } \\
\text { Activa } \\
(\mathrm{kWh})\end{array}$ & $\begin{array}{l}\text { Energía } \\
\text { Reactiva } \\
\text { (kVArh) }\end{array}$ & $\begin{array}{l}\text { Factor de } \\
\text { potencia }\end{array}$ \\
\hline Energía demandada Red Distribución & 24405 & 18500 & 0,8 \\
\hline Energía generada planta FV & 16675 & 0 & 1 \\
\hline $\begin{array}{l}\text { Energía generada banco } \\
\text { compensación }\end{array}$ & & 14904 & \\
\hline Energía resultante & 7730 & 3596 & 0.91 \\
\hline Penalidad & $0 \%$ & & \\
\hline
\end{tabular}

El cálculo del factor de potencia se hizo con base en la ecuación definida según la resolución de la Superintendencia de Electricidad, para determinar si la penalización era eliminada. La tabla 5 muestra un factor 
de potencia menor a 0.9 , lo que corresponde a la penalidad aplicada de $59 \%$, mientras que en la tabla 6 se elimina esta penalidad, lo que tiene sentido ya que luego de aplicar el sistema se consigue elevar el factor de potencia a 0.91 .

Estos datos fueron tomados para la facturación del mes de mayo del año 2019, y el costo del kWh para este período según la Superintendencia de Electricidad fue de 7.26 RD\$/kWh. Según la potencia consumida para este mes el costo por energía eléctrica sería RD \$ 56 119.80, y al aplicar el sistema de compensación de reactivos se estarían ahorrando RD\$ 28871.62 extras a esta tarifa.

\section{Conclusiones}

Los métodos de compensación de reactivos en sistemas fotovoltaicos se adaptan según el perfil de carga y el tamaño de necesidad de compensación. Durante las horas útiles de sol los inversores fotovoltaicos son capaces de inyectar reactivos a la red, disminuyendo la generación de energía activa. Pero, esto disminuye su eficiencia y su vida útil.

Entre los métodos estudiados se compararon sus características para ver el que cumpliera con las especificaciones y se escogió el inversor fotovoltaico conectado a red en caso de que su objetivo sea compensar energía tanto reactiva como activa.

El método aplicado fue el de usar un banco de capacitor automático con filtro desintonizado, y al aplicarlo en el caso práctico detallado en el proyecto se obtuvo un incremento del factor de potencia de un 0.77 a un 0.98 .

El método propuesto en esta investigación para la compensación de reactivos en sistemas fotovoltaicos mejora el factor de potencia de la instalación al mismo tiempo que mantiene la producción de energía eléctrica activa en $\mathrm{kWh} /$ año del inversor fotovoltaico. Además, evita la emisión de aproximadamente $36018 \mathrm{~kg}$ de $\mathrm{CO}_{2}$ anual para la instalación de $138 \mathrm{kWp}$. 


\section{Agradecimientos}

A la empresa de servicios logísticos RODEMSA SAS por permitir el uso de sus instalaciones para realizar las mediciones e implementar las recomendaciones desarrolladas durante la investigación.

\section{Referencias}

ABB. (2016). Corrección del factor de potencia y filtrado de armónicos en las instalaciones eléctricas. Recuperado de https:// library.e.abb.com/public/897462d590876b5fc12571a003bd1e0/ 1TXA007107G0701_CT8.pdf

ABB. (2018). Static Var Compensation (SVC). Recuperado de https:// new.abb.com/facts/static-var-compensation-(svc)

AEMCInstruments. (2016). AEMC 8336 User Manual. Recuperado de https://www.instrumart.com/assets/AEMC-8336-manual.pdf

Ben Youssef, W., Maatallah, T., Menezo, C., \& Ben Nasrallah, S. (2018). Assessment viability of a concentrating photovoltaic/thermal-energy cogeneration system (CPV/T) with storage for a textile industry application. Solar Energy, 159, 841-851. Recuperado de: https:// doi.org/10.1016/j.solener.2017.11.058

Campas, C. N. A., \& Martínez, M. M. (2017, 8-10 Nov. 2017). Interaction between photovoltaic power systems and distribution power systems: Impact on the power factor and energy losses. Paper presented at the 2017 IEEE International Autumn Meeting on Power, Electronics and Computing (ROPEC). Doi: 10.1109/ ROPEC.2017.8261589

Cortez Valladares, N. E., \& Valladares Cordero, H. E. (2017). Estudio de los efectos en el factor de potencia y en el contenido armónico producido por el montaje de un generador fotovoltaico en una instalación eléctrica, (tesis de grado). Universidad de El Salvador. Recuperado de http://ri.ues.edu.sv/15006/

EasyPower. (2019). EasyPower Product Suites. Recuperado de https:// www.easypower.com

Espin, G. C. (2017). Implementación de un Sistema Fotovoltaico y Capacitor para el Ahorro de Energía en Fábrica. Informe Técnico. 
UNIVERSIDAD NACIONAL AUTÓNOMA DE MÉXICO.. Recuperado de http://www.ptolomeo.unam.mx:8080/xmlui/bitstream/handle/132.248.52.100/12800/Informe.pdf?sequence $=1$

Gandhi, O., Rodríguez-Gallegos, C., Reindl, T., \& Srinivasan, D. (2018). Competitiveness of PV Inverter as a Reactive Power Compensator considering Inverter Lifetime Reduction. Energy Procedia, 150, 74-82. Recuperado de: https://doi.org/10.1016/j. egypro.2018.09.005

Gayatri, M. T. L., Parimi, A. M., \& Pavan Kumar, A. V. (2018). A review of reactive power compensation techniques in microgrids. Renewable and Sustainable Energy Reviews, 81, 1030-1036. Recuperado de https://doi.org/10.1016/j.rser.2017.08.006

GSES, T. P. (2016). Power Factor and Grid-Connected Photovoltaics. Recuperado de https://www.gses.com.au/wp-content/uploads/2016/03/ GSES_powerfactor-110316.pdf

Kabir, Y., Mohsin, Y. M., \& Khan, M. M. (2017, 22-24 Feb. 2017). Automated power factor correction and energy monitoring system. Paper presented at the 2017 Second International Conference on Electrical, Computer and Communication Technologies (ICECCT). Doi: 10.1109/ICECCT.2017.8117969

Malatji, E. M., \& Chabangu, B. (2018, 6-7 Dec. 2018). Innovative Method for Power Factor Correction using a Solar Plant as a Source of Reactive Power. Paper presented at the 2018 International Conference on Intelligent and Innovative Computing Applications (ICONIC). Doi: 10.1109/ICONIC.2018.8601214 Miñambres-Marcos, V., Guerrero-Martínez, M. \& Romero-Cadaval, C. (2015). Grid-connected photovoltaic power plants for helping node voltage regulation. IET Renewable Power Generation, 9(3), 236-244. Doi: 10.1049/iet-rpg.2014.0086

Mulolani, F., Armstrong, M., \& Zahawi, B. (2014, 23-25 July 2014). Modeling and simulation of a grid-connected photovoltaic converter with reactive power compensation. Paper presented at the 2014, 9th International Symposium on Communication Systems, Networks \& Digital Sign (CSNDSP). Doi: 10.1109/ CSNDSP.2014.6923954 
Ndiaye, I., Xinhui, W., \& Agamy, M. (2015, 14-19 June 2015). Impact of micro-inverter reactive power support capability in high penetration residential PV networks. Paper presented at the 2015 IEEE 42nd Photovoltaic Specialist Conference (PVSC). Doi: 10.1109/ PVSC. 2015.7356248

Norhasmi, N. N. F., Raveendran, S. K., \& Ramachandaramurthy, V. K. (2018, 7-10 Oct. 2018). Power Factor Control of Solar Photovoltaic Inverter as a Solution to Overvoltage. Paper presented at the 2018 IEEE PES Asia-Pacific Power and Energy Engineering Conference (APPEEC). Doi: 10.1109/appeec.2018.8566582

Organización Latinoamericana de Energía, O. (2013). Recopilación de la Normatividad de Energía Reactiva en otros países e identificación de alternativas regulatorias. Recuperado de http://www.olade.org/ wp-content/uploads/2015/08/Recopilaci\%C3\%B3n-de-la-Normatividad-de-Energ\%C3\%ADa-Reactiva-en-Otros-Pa\%C3\%ADses-e-Identificaci\%C3\%B3n-de-Alternativas-Regu.pdf

Park, A., \& Lappas, P. (2017). Evaluating demand charge reduction for commercial-scale solar PV coupled with battery storage. Renewable Energy, 108, 523-532. Doi: https://doi.org/10.1016/j. renene.2017.02.060

Paulino, Y. (2018). UASD ahorra millones de pesos por reducción en consumo de electricidad. UASD Periódico. Recuperado de https:// www.uasd.edu.do/periodico/index.php/academia/item/2393uasd-ahorra-millones-de-pesos-por-reduccion-en-consumo-deelectricidad

Sarkar, M. N. I., Meegahapola, L. G., \& Datta, M. (2018). Reactive Power Management in Renewable Rich Power Grids: A Review of GridCodes, Renewable Generators, Support Devices, Control Strategies and Optimization Algorithms. IEEE Access, 6, 41458-41489. Doi: 10.1109/ACCESS.2018.2838563

Sayed, M. M. E., El-Ela, A. A. A., \& El-Sehiemy, R. A. (2016, 27-29 Dec. 2016). Effect of photovoltaic system on power quality in electrical distribution networks. Paper presented at the 2016 Eighteenth International Middle East Power Systems Conference (MEPCON). Doi: 10.1109/MEPCON.2016.7837019 
Siecker, J., Kusakana, K., \& Numbi, B. P. (2018, 3-5 April 2018). Economic analysis of photovoltaic/thermal systems with forced circulation under optimal switching control. Paper presented at the 2018 International Conference on the Domestic Use of Energy (DUE). Doi: 10.23919/DUE.2018.8384384

Soni, J., \& Panda, S. K. (2017). Electric Spring for Voltage and Power Stability and Power Factor Correction. IEEE Transactions on Industry Applications, 53(4), 3871-3879. Doi:10.1109/TIA.2017.2681971

Superintendencia de Electricidad, S. (2007). Metodología de Cálculo Factor de Potencia. Superintendencia de Electricidad. Resolución SIE-05-2007.

Uğur, M., Duymaz, E., Göl, M., \& Keysan, O. (2018, 21-25 Oct. 2018). Evaluation of Photovoltaic Systems for Reactive Power Compensation in Low Voltage Power Systems. Paper presented at the 2018 IEEE PES Innovative Smart Grid Technologies Conference Europe (ISGT-Europe). Doi: 10.1109/ISGTEurope.2018.8571808

Vieira, C. R., Silva, K. D. M. e., Garcia, F. R. P., Sampaio, R. F., Leao, R. P. S., Ribeiro, S. D. C., \& Medeiros, E. B. d. (2018, 12-16 May 2018). Analysis of the impacts of the photovoltaic solar generation in the electrical system of distribution. Paper presented at the 2018 Simposio Brasileiro de Sistemas Eletricos (SBSE). Doi: 10.1109/SBSE.2018.8395863

Xavier, L. S., Cupertino, A. F., \& Pereira, H. A. (2018). Ancillary services provided by photovoltaic inverters: Single and three phase control strategies. Computers \& Electrical Engineering, 70, 102121. Doi: https://doi.org/10.1016/j.compeleceng.2018.03.010 